\title{
Parámetros para el diseño de instrumentos en la evaluación del desarrollo de habilidades, competencias y alfabetización informacional
}

\author{
Cinthya Thalia Morales Vitela ${ }^{1}$ \\ Jesús Lau Noriega ${ }^{2}$ \\ Maricela López Ornelas 3
}

\begin{abstract}
Resumen:Este trabajo, de corte empírico y con una medotología descriptiva, recoje la importancia de establecer parámetos para el desarrollo de instrumentos de evaluación de habilidades, competencias y alfaberización informacional, ya que si bien la literatura ha enfatizado en la importancia de la evaluación y diagnóstico de las destrezas y prácticas informativas, la propia fuente presenta además una ausencia este rubro, ya que algunos investigadores reportan la metodología y los resultados del estudio sin proporcionar los instrumentos empleados (LICEA, 2008; LAU, 2017), impidiendo, en este sentido, la oportunidad de que dichos instrumentos puedan ser utilizados, adaptados o replicados por otros académicos. Los resultados de esta investigación, se presentan a través del análisis de los parámetros básicos a considerar en el diseño de instrumentos de evaluación centrados en el área de las habilidades y competencias informativas y mediante la compilación algunos de los instrumentos que evalúan las destrezas y prácticas informativas - recuperados particularmente en repositorios institucionales-.
\end{abstract}

Palabras-clave:Habilidades; Competencias; Alfabetización Informacional; Instrumentos de Evaluación.

\footnotetext{
${ }^{1}$ Licenciada en Psicología, maestranda del Instituto de Investigación y Desarrollo Educativo en la Universidad Autónoma de Baja California, México. E-mail: tmorales@uabc.edu.mx

${ }^{2}$ Doctorado por el Department of Information Studies, University of Sheffield, Inglaterra, Profesor-Investigador de la Universidad Veracruzana, México. E-mail: jesuslau@gmail.com

3Doctora en Comunicación de la Universidad de La Laguna, España. Profesora-Investigador de la Universidad Autónoma de Baja California, México. E-mail: ornelas@uabc.edu.mx
} 


\title{
Parâmetros para concepção de instrumentos na avaliação do desenvolvimento de habilidades, competências e alfabetização informacional
}

\author{
Cinthya Thalia Morales Vitela4 \\ Jesús Lau Noriega 5 \\ Maricela López Ornelas 6
}

\begin{abstract}
Resumo: Este trabalho, de corte empírico e com uma metodologia descritiva, recorre à importância de estabelecer parâmetros para o desenvolvimento de instrumentos de avaliação de habilidades, competências e alfabetização informacional, uma vez que a literatura enfatizou a importância da avaliação e diagnóstico das destrezas e práticas informativas, além disso, a própria fonte apresenta uma ausência deste tema, já que alguns pesquisadores reportam a metodologia e os resultados do estudo sem proporcionar os instrumentos empregados (LICEA, 2008; LAU, 2017), impedindo, neste sentido, a oportunidade de que referidos instrumentos possam ser utilizados, adaptados ou replicados por outros acadêmicos. Os resultados desta investigação, se apresentam através das análises dos parâmetros básicos a considerar a concepção de instrumentos de avaliação centrados nas áreas das habilidades e competências informativas e mediante a compilação alguns dos instrumentos que avaliam as destrezas e práticas informativas -, recuperados particularmente nos documentos institucionais-.
\end{abstract}

Palavras-chave:Habilidades; Competência; Alfabetização Informacional; Instrumentos de Avaliação.

4Licenciada em Psicología, mestranda no Instituto de Investigación y Desarrollo Educativo na Universidad Autónoma de Baja California, México. E-mail: tmorales@uabc.edu.mx

5Professor na Universidad Veracruzana, México. Doutor pela Universidade de Sheffield na Inglaterra. E-mail: jesuslau@gmail.com

${ }^{6}$ Professora na Universidad Autónoma de Baja California, México . Doutora em comunicação pela Universidade de La Laguna, na Espanha. E-mail: ornelas@uabc.edu.mx 


\section{Introducción}

Para atender los requerimientos que nos demanda el entorno informacional en el que nos encontramos inmersos, surge la necesidad de transmitir en los individuos destrezas y prácticas que los hagan más ávidos en el uso eficaz de la información (AREA, 2014). Sobre todo, porque en las últimas décadas se ha incrementado la complejidad en la apropiación y transmisión del conocimiento como derivación de las nuevas tecnologías, los servicios y los medios de comunicación (BAWDEN, 2002; BAWDEN y LYN, 2017). Debido a este fenómeno informativo se precisa la necesidad de adquirir habilidades y competencias con el fin de dar respuesta a distintos problemas y nuevas necesidades (BIELBA, MARTÍNEZ, HERRERA y RODRÍGUEZ, 2015). En este sentido, estas destrezas -a las que se hace mención- han sido estudiadas desde diversas posturas, por lo que concebir un concepto único y claro de cómo referirse a ellas resulta complejo y por ende, necesario de explicar.

Al respecto, a través de la literatura consultada se han encontrado diferentes formas de aludir a estas prácticas, algunas de ellas son: Alfabetización Informacional -ALFIN-, posicionándose como la de mayor presencia en países como España y Colombia, en donde sus principales expositores son Licea de Arenas, Gómez, y Valles (2009); Pineda, et al., (2012); Quevedo (2014); Uribe (2014); Area, (2014) y García (2015); mientras que en países como México, el vocablo mayormente aplicado ha sido Desarrollo de habilidades informativas u informacionales, también denominadas Competencias Informacionales - CI-, término que ha ido referenciado especialmente en Cuba y México por investigadores como Lau y Cortés (2009), Sánchez (2008); Catts y Lau (2009); Villanueva y Casas(2010); Area y Pessoa (2012); Area, Gutiérrez y Vidal (2012); Lau, et al. (2015); Bielba, et al. (2015); otro planteamiento es Desarrollo de Habilidades Informativas - DHI-, con presencia particularmente en México y España, y promovido por autores como Domínguez y Ocampo (2008); además del Ministerio de Educación (2013); por último, pero no menos importante, la Alfabetización Informacional Digital -AID_, cuya visibilidad ha tenido menor presencia, donde sus principales referecian hacen mención en países 
como Costa Rica y Cuba, por Álvarez, Gisbert yGónzalez(2013); Fernández, Díaz y Delgado (2013); respectivamente-. Si bien existen combinaciones o fusiones entre estas variantes, la mayor preocupación, es que son aplicadas indistintamente a pesar de tener una connotación diferente. Si bien no es el objetivo de esta investigación, es responsable exponer que existe la necesidad de una convergencia terminológica.

Por consiguiente, la polisemia del concepto no es la única diversificación que existe en cuanto al estudio de estas destrezas o prácticas informacionales, sino que también varía su análisis en las numerosas áreas de aplicación, como son, su incorporación en las instituciones, en los programas académicos, en las prácticas docentes y en las prácticas de los estudiantes - esta última identificada como la menos explorada-. Por lo que resulta importante, además de la propia transferencia de tales conocimientos, contar con los procesos de evaluación y diagnóstico específicos de cada ámbito. Ya que la valoración en su conjunto proporciona información necesaria para contribuir en la mejora, planificación, desarrollo y control de las estrategias diseñadas para optimizar las destrezas informacionales (MENESES y PINTO, 2011).

Si bien la literatura ha enfatizado en la importancia de la evaluación y diagnóstico de las destrezas y prácticas informativas, se observa mayormente que las investigaciones enfocadas a este campo resultan incompletas,puesto que algunos investigadores reportan la metodología y los resultados del estudio sin proporcionar los instrumentos empleados (LICEA, 2008;LAU, comunicación personal, 25 de agosto del 2017). Impidiendo, en este sentido, la oportunidad de que dichos instrumentos puedan ser utilizados, adaptados, replicados o sirvan de inspiración a otros investigadores, existiendo una carencia de acceso a las herramientas para la valoración de las competencias y habilidades informativas de diversos estudios. Por lo que el presente artículo tiene por objetivo aportar en esta área los estudios que han compartidos sus instrumentos, y ofrecer un análisis de los puntos importantes a considerar en los instrumentos de evaluación enfocados al área de habilidades y competencias informativas. 
Bajo esta perspectiva, en el documento se pretende primeramente hacer una breve explicación de la importancia de la evaluación; proveer una definición de instrumento y mencionar los tipos de herramientas relevantes en este campo de estudio, así como algunas de sus características y una serie de consideraciones y recomendaciones en el desarrollo de los instrumentos de medición. Para posteriormente esbozar el panorama que se reporta en la literatura y finalmente presentar una compilación de algunos de los instrumentos utilizados para evaluar la alfabetización informacional, las competencias y habilidades informacionales, y la alfabetización informacional digital - de acuerdo al enfoque del autor-. Esto, con el objetivo de crear un documento que sea de ayuda para investigadores interesados a esta área.

\section{La importancia de la evaluación}

Sin entrar a detalle con la amplitud propia del concepto y su impacto en los procesos educativos - ya que no es el punto focal en este análisis- en los últimos años han surgido diversas concepciones sobre la evaluación, por lo que también exigen un acuerdo terminológico (HAMODI, LÓPEZ-PASTOR y LÓPEZ-PASTOR, 2015). Este apartado retoma de forma puntualla Ley del Instituto Nacional para la Evaluación de la Educación, publicada en México por el Diario Oficial de la Federación (DOF, 2013) - aún vigente en la Nueva Reforma Educativa-, que hace mención en el artículo 7 que los fines de la evaluación consisten en:

I. Contribuir en la mejora de la calidad de la educación; II. Contribuir en la formulación de políticas educativas y el diseño e implementación de los planes y programas [...]; III. Ofrecer información sobre el grado de cumplimiento de los objetivos de mejora establecidos por las autoridades educativas; IV. Mejorar la gestión escolar y los procesos educativos [...] y por último V. Fomentar la transparencia y la rendición de cuentas [...] (DOF, 2013, p.22)

En este sentido se reafirma la postura de Meneses y Pinto (2011), quienes mencionan que, asegurar el uso eficaz de la información, resulta insuficiente cuando únicamente se realiza la transferencia de prácticas, sin antes contar con un proceso de evaluación y diagnóstico. Ya que ambos procesos, proporcionan la información 
necesaria para mejorar y auxiliar en la planificación, desarrollo y control de los planes y estrategias, diseñados para incorporar las competencias en lo usuarios. De modo que, se puede decir que la evaluación siempre será determinada por los objetivos que se busquen alcanzar y es importante visualizarla como un continuo de mejora evaluación y diagnóstico- en donde ningún proceso es más importante que otro (LICEA, 2008).

\subsection{Técnicas e instrumentos: Diferencias}

La revisión de la literatura especializada permite comprobar que actualmente es difícil encontrar diferenciaciones claras sobre los conceptos de "medios", "técnicas" e "instrumentos" de evaluación del aprendizaje. También se observa cierta confusión en el uso de términos como: instrumentos, herramientas, técnicas, recursos, métodos, enfoques, dispositivos y procedimientos de evaluación (HAMODI; LÓPEZ-PASTOR y LÓPEZ-PASTOR, 2015, p. 158).

Es importante hacer un pequeño paréntesis para mencionar que existe una diferencia entre técnicas e instrumentos, ya que en ocasiones suele confundirse. De acuerdo a Rodríguez y Valldeoriola (2009), las técnicas hacen referencia a las formas en las que se recoge la información, como el análisis documental, la autobiografía, las historias de vida, etc. Mientras que los instrumentos, son herramientas concretas que permiten llevar a la práctica la obtención de la información, los cuales se caracterizan por asumir una entidad propia y ser poco flexibles.

Dentro de la diversidad de definiciones al respecto, se considera relevante la propuesta de Hernández, Collado y Lucio (2010), quienes señalan que un instrumento de medición, "es un recurso que utiliza el investigador para registrar información o datos sobre las variables que tiene en mente” (p. 200). Así mismo, mencionan que el principal propósito de estas herramientas, es recabar los datos observables para poder representar los conceptos o variables del objeto de estudio. Esta concepción es reforzada por Villanueva (2014), quien menciona que las herramientas permiten la recuperación de la información y añade, que es necesario 
estimular la presencia de lo que se pretende evaluar para obtener los resultados esperados.

Debido a esto, es fundamental otorgar la importancia correspondiente a la elaboración y uso de los instrumentos de medición, tomando en consideración los requisitos específicos con los que debe contar. Criterios, que de acuerdo a la literatura sobre metodología de la investigación, son: la confiabilidad, validez y objetividad, esto quiere decir, 1) que el instrumento produzca resultados consistentes y coherentes; 2) que mida realmente lo que pretende medir, tanto en contenido, criterio y constructo y, 3) que evite los sesgos y preferencias de los investigadores que lo manejan, a través de un acuerdo consensual de lo que se observa (KERLINGER y LEE, 2002; y HERNÁNDEZ; COLLADO y LUCIO, 2010).

En este aspecto, además de tomar en cuenta los tres factores señalados, es esencial diferenciar también entre los tipos de herramientas que existen, esto, con la finalidad de hacer la elección adecuada para responder a los objetivos y estrategias de evaluación perseguidas (RODRÍGUEZ y VALLDEORIOLA, 2009). Bajo esta perspectiva, se presentan a continuación distintos tipos de instrumentos de medición considerados pertinentes para los objetivos de este trabajo.

\subsection{Tipos de instrumentos}

Como se indicó previamente, los instrumentos sirven para la recolección de la información y a diferencia de las técnicas, son más estrictos desde su construcción hasta su uso, gracias a esto se puede decir, que son las herramientas lo que permite el análisis de lo que se quiere evaluar (RODRÍGUEZ y VALLDEORIOLA, 2009). Así pues, es importante tomar en cuenta que existen diferentes tipos de instrumentos de medición, tanto para los enfoques cualitativos, como para enfoques cuantitativos y cada uno cuenta con características específicas para su elaboración.

Entre los instrumentos utilizados en el enfoque cualitativo, se encuentran: la observación participante, la entrevista a profundidad y los documentos personales, por referir algunos. Mientras que en el enfoque cuantitativo -objeto de estudio en 
este artículo-, se identifican los cuestionarios, las pruebas objetivas, la observación sistemática y la entrevista estructurada. Mismos que a continuación se definen brevemente:

1. Cuestionario:Consiste en un conjunto de preguntas respecto a una o más variables a medir, este puede tener preguntas abiertas o cerradas. En ocasiones también se refieren a estos como escalas, la cuales, de acuerdo a Kerlinger y Lee (2002), pueden ser de actitud o de valores. Su diseño se basa en los hechos que interesan al estudio de investigación (MUÑOZ, 2003).

2. Pruebas objetivas:Es un procedimiento sistemático en donde se presenta al individuo un conjunto de estímulos en donde responde con valores numéricos. Existen diversos tipos: de inteligencia, aptitud, de rendimiento y de personalidad (KERLINGER y LEE, 2002).

3. Observación sistemática:Recolecta información respecto a conductas y procesos, en donde se evalúan hechos, comportamientos y no hay mediciones indirectas, se utilizan listas de cotejo y registros de control (RODRÍGUEZ y VALLDEORIOLA, 2009).

4. Entrevista estructurada o estandarizada: Emplea inventarios de entrevistas elaborados cuidadosamente, permite explorar el contexto y las respuestas pueden ser más precisas (HERNÁNDEZ, COLLADO y LUCIO, 2010).

Si bien existen otros instrumentos utilizados en el enfoque cuantitativo, para este documento, se consideraron las herramientas empleadas en las investigaciones que evalúan las competencias y habilidades informacionales entendidas también como alfabetización informacional. Con base en lo anterior y para precisar, se identificó que los cuestionarios y las escalas son los instrumentos mayormente aplicados por los autores, por lo que basados en este antecedente, se presentan algunas consideraciones para la realización de dichos instrumentos. 


\subsection{Cuestionarios y escalas: factores importantes}

A la hora de elaborar cualquier instrumento de medición es preciso llevar a cabo ciertas fases que determinarán la eficiencia de la herramienta, de tal modo, los cuestionarios y escalas no son la excepción. Por lo que, en este apartado se exponen las primeras siete fases en el proceso de la construcción de un instrumento de medición - por considerarse las más relevantes en el desarrollo de cualquier herramienta - derivadas del trabajo de Hernández, Collado y Lucio (2010):

- Redefinicionesfundamentales: Se reevalúan las variables de investigación, se delimita el lugar, el momento, el tipo y el propósito de la recolección de datos y las definiciones operacionales.

- Revisiónenfocadaalaliteratura: Se buscan los instrumentos y/o sistemas de medición utilizados en otros estudios, que asemejen las variables de estudio, con la finalidad de identificar herramientas útiles en la propia investigación.

- Identificacióndeldominiodevariablesamedirysusindicadores: Se identifican componentes, dimensiones y factores que integran la variable.

- Pruebapiloto: Se aplica el instrumento a una pequeña muestra para comprobar la pertinencia y eficacia, así como, conocer las condiciones y procedimientos a aplicar.

- Construccióndelinstrumento: Se crean los ítems, reactivos o categorías del instrumento y se decretan los niveles de medición y la codificación.

- Tomadedecisionesclave: Se decide por, 1) utilizar un instrumento elaborado, adaptado o propio; 2) en caso de elaborar uno, se decide su tipo y formato, y por último 3) se establece el contexto de aplicación.

- Elaboracióndelaversiónfinal: Se revisa el instrumento con el objetivo de implementar los cambios necesarios y finalmente construir la versión definitiva.

Finalmente, para que cualquier instrumento de evaluación logre el éxito esperado -medir realmente lo que se quiere medir-, es necesario contar con dos elementos; emplear la escala de medición adecuada, y analizar que exista relación 
entre los reactivos, indicadores, variables, objetivos y/o la hipótesis de la investigación. Esto, también es de suma importancia considerarlo a la hora de realizar adaptaciones o cuando se utilizan instrumentos ya establecidos.

En virtud de ello, se considera que una de las mayores aportaciones de este trabajo, consiste precisamente en proveer una serie de recomendaciones que permitan a los investigadores, atender diferentes características en el diseño de los instrumentos de medición. Las cuales se señalan en base a las perspectivas de Muñoz (2003),Álvarez, Gisbert yGónzalez (2013) y Lau (2017), mismas que se clasificaron bajo tres rubros: formato, preguntas y respuestas.

En el formato:

- Colocar un título que refleje lo que mide el instrumento.

- Entablar un diálogo cordial con el participante.

- Contar con instrucciones claras y precisas.

- Mencionar el anonimato y confidencialidad (cuando estos datos no sean necesarios).

- Verificar que se cuente con el espacio suficiente para anotar la respuesta.

- Definir el formato de aplicación: digital o lápiz y papel.

En las preguntas:

- Precisar el tipo de cuestionario o encuesta: estructurado, no estructurado o semiestructurado.

- Establecer el tipo de preguntas: cerradas, abiertas o mixtas.

- Plantear la clase de pregunta: de hechos, opinión, actitudes, motivaciones, sentimientos, o conocimientos.

- Categorizar los apartados (en caso de tenerlos).

- Evitar que sea muy extenso. 
- Enumerar todas las preguntas.

- Realizar preguntas claras y concisas.

- Omitir preguntas dobles o innecesarias.

- Evitar preguntar información que ya esta disponible en otras fuentes

- Prevenir que la redacción de la pregunta no incida en la respuesta.

- Unificar la redacción de las preguntas, en cuanto a la narrativa, verbos y tiempos.

- Variar en la redacción de las preguntas, para evitar la monotonía.

En las respuestas:

- Fijar el nivel de medición de acuerdo al tamaño de la muestra.

- Concretar si se utilizará alguna escala de tipo Likert (de acuerdo, de frecuencia, de uso, etc.).

- En el caso de las preguntas cerradas, comprobar que éstas proporcionen la respuesta requerida

- Establecer de forma precisa en las instrucciones, la forma de responder el cuestionario.

- Evitar la ambigüedad en las preguntas abiertas, para que las respuestas sean concretas.

- Unificar el estilo de respuesta en las preguntas cerradas.

Así mismo, es importante señalar que aunque dichas recomendaciones no son las únicas a considerar, en primera instancia sí proponen aspectos relevantes a tomar en cuenta al momento del primer bosquejo de un cuestionario.Y éstas deberán replantearse en diversas ocasiones, conforme a la etapa del proceso de construcción en el que se encuentre el instrumento, es decir, como resultado de las revisiones propias y de los pares académicos, posterior a la aplicación del pilotaje y finalmente en la versión definitiva. 
Hasta este punto, el presente artículo ha tenido el propósito de bosquejar la importancia de la evaluación y los instrumentos de medición -haciendo especial énfasis en el cuestionario y la escala-, proponiendo en este sentido, una serie de recomendaciones que se deben abordar a la hora de realizar cualquier herramienta de evaluación. A continuación, se expone el panorama que muestra la literatura en cuanto a los instrumentos utilizados para evaluar la alfabetización informacional, las competencias y habilidades informacionales así como la alfabetización informacional digital.

\section{Instrumentos de evaluación en DHI, ALFIN, CI y AID}

Como se mencionó al inicio, existen diferentes posturas para referirse a las destrezas necesarias en la interacción eficaz del usuario de la información, y a su vez, también distintos ámbitos en los que se incorporan estas prácticas. Estas son, la integración de dichas competencias en las instituciones, en los programas académicos, en las prácticas docentes y en las prácticas de los estudiantes, lo que vuelve inevitable que se establezcan instrumentos particulares para hacer las evaluaciones correspondientes en cada ámbito. Bajo esta línea, se plantean a continuación las aportaciones consideradas más significativas en cuanto a los instrumentos utilizados para evaluar las prácticas de alfabetización informacional, las competencias y habilidades informacionales, así como la alfabetización informacional digital, en sus numeros ámbitos. Esto, con la intención de proporcionar un panorama acerca de las tendencias en las investigaciones sobre el tema y a su vez, suministrar a los interesados las características de los instrumentos de evaluación que persisten en la materia.

Algunos de los ejemplos más representativos en el campo de la evaluación de las habilidades y competencias informativas en Estados Unidos son, el proyecto Standardized Assessment of Information Literacy Skill (SAILS), el proyecto Information Skills y el proyecto The Threshold Achievement Test for Information 
Literacy (TATIL). SAILS (2008), fue establecido por la Universidad de Kent y retomado por la Association of University and Research Libraries (ACRL), mientras que el proyecto iSkills (2008), fue creado por el Educational Testing Service and Information and Communications Technology(ETS-ITC), el mismo que genera diversos tests, entre el ellos el TOEFL (Test of English as Foreign Language) y por último el instrumento TATIL (2017), recientemente desarrollado por Carrick Enterprises, una empresa encargada de la evaluación del rendimiento estudiantil. Los primeros dos proyectos se encuentran asentados bajo las "Normas sobre aptitudes para el acceso y uso de la información en la enseñanza superior: estándares, indicadores de rendimiento y resultados", establecidas por la ACRL en el año 2000 (LAU, MACHIN, GARATE y TAGLIAPIETRA, 2016), mientras que el proyecto TATIL (2017), se encuentra fundamentado en el "Marco para la Alfabetización de la Información para la Educación Superior" y en las expectativas establecidas por las agencias de acreditación de la nación de la ACRL.

Estos proyectos, se crearon para ofrecer a bibliotecarios, educadores y administradores, una mejor comprensión sobre las habilidades de alfabetización informacional en los estudiantes y las áreas de mejora en los que se puede incidir, para de esta manera, fortalecer dichos aspectos en la comunidad universitaria (MENESES y PINTO, 2011; RADCLIFF, 2017).

Por otro lado en el ámbito iberoamericano, encontramos que si bien existen investigaciones acerca de la evaluación de la alfabetización informacional, las habilidades y competencias informativas y la alfabetización informacional digital, estas se caracterizan por no presentar los instrumentos utilizados en dichos estudios, lo que ha generado una escasez en la literatura. Como resultado de lo anterior, este trabajo plantea una revisión de la literatura referente a investigaciones enfocadas al diseño, adaptación y aplicación de instrumentos de evaluación sobre el tema, con el propósito de proveer una relación en cuanto a las similitudes en el tipo de evaluación, sus características y el objeto de estudio en los instrumentos (véase Tabla 1), que pueda servir a otros investigadores de este campo. 
Tabla 1 - Instrumentos de evaluación de ALFIN, CI, DHI y AID en Iberoamérica.

\begin{tabular}{|c|c|c|c|c|}
\hline Autores & País & $\begin{array}{c}\text { Tipo de } \\
\text { evaluación }\end{array}$ & Objeto de estudio & Características \\
\hline 1. URIBE, T. (2008) & Colombia & $\begin{array}{l}\text { Competencias } \\
\text { informacionales }\end{array}$ & $\begin{array}{l}\text { Prácticas en } \\
\text { estudiantes } \\
\text { universitarios }\end{array}$ & $\begin{array}{ll}\checkmark & \text { Cuestionario de } \\
& \text { autoevaluación } \\
\checkmark & \text { Basado en criterios } \\
\checkmark & \text { Seis apartados } \\
\checkmark & 23 \text { reactivos }\end{array}$ \\
\hline $\begin{array}{l}\text { 2. CASTAÑNEDA - } \\
\text { PEÑA, GONZÁLEZ, } \\
\text { MARCIALES, } \\
\text { BARBOSA y } \\
\text { BARBOSA (2010) }\end{array}$ & Colombia & $\begin{array}{l}\text { Competencias } \\
\text { informativas }\end{array}$ & $\begin{array}{l}\text { Prácticas en } \\
\text { estudiantes } \\
\text { universitarios }\end{array}$ & $\begin{array}{ll}\checkmark & \text { Instrumento } \\
& \text { observacional } \\
\checkmark & \text { Cuatro secciones } \\
\checkmark & \text { Valoradas en categorías y } \\
& \text { sub categorías } \\
\checkmark & \text { Caracterización de } \\
& \text { perfiles }\end{array}$ \\
\hline $\begin{array}{l}\text { 3. BIELBA, } \\
\text { MARTÍNEZ, } \\
\text { HERRERA } \\
\text { yRODRÍGUE (2015) }\end{array}$ & España & $\begin{array}{l}\text { Competencias } \\
\text { informacionales }\end{array}$ & $\begin{array}{l}\text { Prácticas en } \\
\text { estudiantes en } \\
\text { educación } \\
\text { secundaria }\end{array}$ & $\begin{array}{ll}\checkmark & \text { Cuestionario de } \\
& \text { valoración de los } \\
& \text { indicadores. } \\
\checkmark & \text { Cuatro dimensiones } \\
\checkmark & \text { Tabla de especificaciones } \\
& \text { de las dimensiones. } \\
\checkmark & 42 \text { indicadores } \\
\checkmark & \text { Validación por expertos }\end{array}$ \\
\hline $\begin{array}{l}\text { 4. RODRÍGUEZ- } \\
\text { CONDE, OLMOS y } \\
\text { MARTÍNEZ-ABAD } \\
(2012)\end{array}$ & España & $\begin{array}{l}\text { Competencias } \\
\text { informacionales }\end{array}$ & $\begin{array}{l}\text { Prácticas en } \\
\text { estudiantes } \\
\text { universitarios }\end{array}$ & $\begin{array}{ll}\checkmark & \text { Escala de actitud } \\
\checkmark & \text { Tres secciones } \\
\checkmark & 26 \text { reactivos }\end{array}$ \\
\hline $\begin{array}{l}\text { 5. ÁLVAREZ, } \\
\text { GISBERT y } \\
\text { GÓNZALEZ (2013) }\end{array}$ & España & $\begin{array}{l}\text { Alfabetización } \\
\text { informacional digital }\end{array}$ & Practicas docentes & $\begin{array}{ll}\checkmark & \text { Cuestionario de } \\
& \text { autoevaluación } \\
\checkmark & 57 \text { reactivos } \\
\checkmark & \text { Preguntas de simulación } \\
& \text { y de actitudes }\end{array}$ \\
\hline $\begin{array}{l}\text { 6. LAU, GARATE y } \\
\text { OSUNA (2015) }\end{array}$ & México & $\begin{array}{l}\text { Competencias } \\
\text { informativas }\end{array}$ & Prácticas docentes & $\begin{array}{ll}\checkmark & \text { Cuestionario en línea de } \\
& \text { autoevaluación } \\
\checkmark & \text { Datos generales } \\
\checkmark & \text { Cinco secciones } \\
\checkmark & 17 \text { reactivos } \\
\checkmark & \text { Cuestionario mixto }\end{array}$ \\
\hline $\begin{array}{l}\text { 7. WILLIAM, P. } \\
\text { (2017) }\end{array}$ & Hondu-ras & $\begin{array}{l}\text { Competencias en } \\
\text { información }\end{array}$ & $\begin{array}{l}\text { Prácticas en } \\
\text { estudiantes }\end{array}$ & $\begin{array}{ll}\checkmark & \text { Cuestionario de } \\
& \text { autoevaluación } \\
\checkmark & \text { Cinco apartados } \\
\checkmark & 55 \text { reactivos } \\
\checkmark & \text { Cuestionario mixto }\end{array}$ \\
\hline
\end{tabular}




\begin{tabular}{|c|c|c|c|c|}
\hline Autores & País & $\begin{array}{c}\text { Tipo de } \\
\text { evaluación }\end{array}$ & Objeto de estudio & Características \\
\hline $\begin{array}{l}\text { 8. BONILLA, } \\
\text { CONTRERAS, } \\
\text { OSUNA, ROBLES, } \\
\text { MARTÍNEZ, } \\
\text { GUTIÉRREZ, } \\
\text { TAGLIAPRIETA y } \\
\text { GARATE (2012) }\end{array}$ & México & $\begin{array}{l}\text { Habilidades } \\
\text { informativas }\end{array}$ & $\begin{array}{l}\text { Prácticas en } \\
\text { estudiantes } \\
\text { universitarios }\end{array}$ & $\begin{array}{ll}\checkmark & \text { Cuestionario en línea de } \\
& \text { autoevaluación } \\
\checkmark & \text { Datos generales } \\
\checkmark & \text { Cinco secciones } \\
\checkmark & 17 \text { reactivos } \\
\checkmark & \text { Cuestionario mixto }\end{array}$ \\
\hline 9. BONILLA (2017) & México & $\begin{array}{l}\text { Competencias y } \\
\text { Habilidades } \\
\text { Informativas }\end{array}$ & $\begin{array}{l}\text { Prácticas en } \\
\text { estudiantes } \\
\text { universitarios }\end{array}$ & $\begin{array}{ll}\checkmark & \text { Cuestionario de } \\
& \text { autoevaluación } \\
\checkmark & \text { Tres apartados } \\
\checkmark & 42 \text { reactivos } \\
\checkmark & \text { Cuestionario mixto } \\
\checkmark & \text { Adaptación de rubrica } \\
\checkmark & \text { Para evaluar trabajos } \\
& \text { escritos por los docentes } \\
\checkmark & \text { Basada en criterios } \\
\end{array}$ \\
\hline $\begin{array}{l}\text { 10. FERNÁNDEZ } \\
\text { (2017) }\end{array}$ & México & $\begin{array}{l}\text { Competencias } \\
\text { informativas }\end{array}$ & Practicas docentes & $\begin{array}{ll}\checkmark & \text { Cuestionario de } \\
& \text { autoevaluación } \\
\checkmark & \text { Aplicado en línea o papel } \\
\checkmark & \text { tres apartados } \\
\checkmark & 27 \text { reactivos } \\
\checkmark & \text { Cuestionario mixto } \\
\end{array}$ \\
\hline $\begin{array}{l}\text { 11. AGUSTIN (EN } \\
\text { PRENSA) }\end{array}$ & México & $\begin{array}{l}\text { Competencias } \\
\text { informativas }\end{array}$ & $\begin{array}{l}\text { Prácticas en } \\
\text { estudiantes } \\
\text { universitarios }\end{array}$ & $\begin{array}{ll}\checkmark & \text { Datos generales } \\
\checkmark & \text { Cinco secciones } \\
\checkmark & 50 \text { reactivos } \\
\checkmark & \text { Cuestionario mixto }\end{array}$ \\
\hline $\begin{array}{l}\text { 12. AVELAR, M. } \\
\text { (2011) }\end{array}$ & México & $\begin{array}{l}\text { Herramientas de } \\
\text { ALFIN }\end{array}$ & $\begin{array}{l}\text { Prácticas en } \\
\text { estudiantes y } \\
\text { docentes }\end{array}$ & $\begin{array}{ll}\checkmark & \text { Cuestionario de opinión } \\
\checkmark & \text { Cuatro apartados } \\
\checkmark & 12 \text { reactivos } \\
\checkmark & \text { Cuestionario mixto }\end{array}$ \\
\hline $\begin{array}{l}\text { 13. NOLASCO, } \\
\text { P.(2017) }\end{array}$ & México & $\begin{array}{l}\text { Tecnologías de la } \\
\text { información y } \\
\text { comunicación }\end{array}$ & $\begin{array}{l}\text { Incorporación en } \\
\text { las instituciones de } \\
\text { educación superior }\end{array}$ & $\begin{array}{ll}\checkmark & \text { Cuestionario aplicado a } \\
& \text { administrativos, docentes } \\
& \text { y estudiantes } \\
\checkmark & \text { Cinco secciones } \\
\checkmark & 10 \text { reactivos } \\
\checkmark & \text { Preguntas cerradas }\end{array}$ \\
\hline 14. SALAS, R. (2017) & México & $\begin{array}{l}\text { Competencias } \\
\text { informacionales }\end{array}$ & $\begin{array}{l}\text { Prácticas en } \\
\text { estudiantes }\end{array}$ & $\begin{array}{ll}\checkmark & \text { Cuestionario de } \\
& \text { autoevaluación } \\
\checkmark & \text { Dos secciones } \\
\checkmark & 30 \text { reactivos } \\
\checkmark & \text { Cuestionario mixto }\end{array}$ \\
\hline $\begin{array}{l}\text { 15. MORALES- } \\
\text { VITELA Y LÓPEZ- } \\
\text { ORNELAS, (2017) }\end{array}$ & México & $\begin{array}{l}\text { Competencias } \\
\text { informacionales }\end{array}$ & $\begin{array}{l}\text { Prácticas en } \\
\text { estudiantes }\end{array}$ & $\begin{array}{ll}\checkmark & \text { Cuestionario de } \\
\text { autoevaluación } \\
\checkmark & \text { Cinco secciones } \\
\checkmark & 23 \text { reactivos }\end{array}$ \\
\hline
\end{tabular}




\begin{tabular}{|l|l|l|l|l|}
\hline Autores & País & $\begin{array}{c}\text { Tipo de } \\
\text { evaluación }\end{array}$ & Objeto de estudio & Características \\
\hline & & & & $\checkmark$ Preguntas en escala likert \\
\hline
\end{tabular}

Fonte: Desenvolvido pelos autores

Es importante señalar que de las 15 investigaciones registradas en el Cuadro 1, únicamente los trabajos de Uribe (2008); Castañeda-Peña et al. (2010); Bielba et al. (2015); Rodríguez-Conde, Olmos y Martínez-Abad (2012); Álvarez, Gisbert yGónzalez (2013); Lau, Garate y Osuna (2015); y Morales-Vitela y LópezOrnelas (2017), se encuentran actualmente publicados, y si bien, no son los únicos estudios realizados en la evaluación de las prácticas y destrezas en el uso de la información, fueron consideradas, dada su importancia por presentar los instrumentos de evaluación en los temas analizados. En cuanto a las ocho investigaciones restantes, entre las características que comparten, destaca que son trabajos de posgrado aún no publicados, lo que los convierte en investigaciones de difícil alcance y de escasa visibilidad en el ámbito académico.

La sociedad de la información, que algunos llaman también en forma más elevada sociedad del conocimiento (GARCÍA, 2015) en la que vivimos en el siglo XXI, nos exige el desarrollo de habilidades, prácticas y destrezas útiles en el uso eficaz de la información, lo que representa la necesidad de establecer instrumentos y proceso que permitan su evaluación constante, con la finalidad de mejorar dichas competencias en los ámbitos correspondientes, además de requerir la evaluación propia de los instrumentos que las miden. De manera que esta dinámica cíclica llamada "evaluación", se vuelve complicada, particularmente - en este tema-, ya que existen pocos instrumentos registrados en la literatura científica, por lo que es importante que los investigadores, además de compartir los resultados de sus estudios, se interesen en proporcionar la estructura concerniente a los instrumentos empleados en sus trabajos. Pues de esta manera, se generan mayores oportunidades 
dentro de la comunidad académica que estudia este tipo de competencias, entre ellos, utilizar, adaptar o replicar sus instrumentos en trabajo propios.

\section{Consideraciones finales}

A manera de reflexión, se reconoce que el análisis presentado en esta investigación, no facilita la sistematización conceptual o el uso de indicadores entre las Competencias Informacionales - CI-, el Desarrollo de Habilidades Informativas -DHI-, o la Alfabetización Informacional Digital -AID-, pues cada término trae consigo una serie de concepciones, dimensiones y categorías que varían entre los autores según el objeto de estudio, por tanto, la concordancia terminológica no es un objetivo en esta investigación.

En la misma línea, se reconoce la existencia de investigaciones científicas enfocadas al diseño e implementación de instrumentos para la evaluación de habilidades y competencias informativas, del mismo modo se identifica, la escasez de instrumentos validados y publicados para dichas áreas (BIELBA, et al. 2015, p. 126).

Si bien se está consciente de que el estudio de los 14 trabajos constituye una limitación en los resultados, pues sin lugar a duda existe una riqueza inmensa sobre este tema, lamentablemente son pocos los trabajos que incluyen los instrumentos en los artículos, y en consecuencia, al no estar publicados, son invisibles para los sistemas actuales de búsqueda.

Bajo esta perspectiva, este estudio considera la necesidad e importancia de publicar e incluir en las investigaciones los instrumentos diseñados para la evaluación de competencias informacionales, habilidades informativas y alfabetización informacional, con la finalidad de suministrar tales aportaciones a la comunidad académica, para que puedan generar conocimiento sobre bases sólidas, y ser utilizados, adaptados y/o replicados, lo que en resumen se traduce, como contribución a un proceso formativo para investigadores noveles.

Conjuntamente, es significativo incentivar a nuevos investigadores a crear sus propios instrumentos utilizando como guía las recomendaciones aportadas en este documento, teniendo como finalidad su publicación y, bajo esta línea, establecer 
una generación de expertos sensibilizados en proporcionar y difundir el conocimiento en la comunidad científica.

Precisamente por lo expresado en el párrafo anterior, uno de los objetivos en esta investigación, fue proveer un tipo de guía de aprendizaje sobre los parámetros más importantes a considerarse en el diseño de instrumentos de evaluación establecidos en el área de las habilidades y competencias informativas, con el fin de ofrecer un recorrido elemental, que facilite el proceso de aprendizaje de quienes necesiten incursionar-en un nivel básico-, y proveer una serie de estrategias para ayudarlos a avanzar en un primer bosquejo de un instrumento para evaluar este tipo de información.

Por lo que el objetivo principal de este estudio es aportar al área de las investigaciones que han compartidos sus instrumentos, y ofrecer un análisis de los puntos importantes a considerar en los instrumentos de evaluación enfocados al área de habilidades y competencias informativas.

\section{Referências}

ÁLVAREZ, Juan; GISBERT, Marcé y GÓNZALEZ, Juan. Validación de un cuestionario para medir el nivel de alfabetización informacional digital del profesorado de educación secundaria de todo el Estado español. 2013. EDUTEC. Disponível em:

https://www.uned.ac.cr/academica/edutec/memoria/ponencias/alvarez gisbert 99 .pdf Acesso em: 22 abr. 2018.

AMOR, Ma y DELGADO, Águeda. De la competencia digital y audiovisual a la competencia mediática: dimensiones e indicadores. Lumina v. 11, n. 1, p. 1-21, 2017. Disponível em:https://lumina.ufff.emnuvens.com.br/lumina/article/view/718Acesso em: 22 abr. 2018.

AREA, Manuel. La alfabetización digital y la formación de la ciudadanía del siglo XXI. Revista Integra Educativa, n. 7, v. 3. p.1-13, 2014. Disponível em: http://scielo.org.bo/pdf/rieiii/v7n3/v7n3 a02.pdfAcesso em: 22 abr. 2018.

AREA, Manuel; GUTIÉRREZ, Alfonso y VIDAL, Fernando. Alfabetización digital y competencias informacionales. Madrid: Ariel y Fundación Telefónica, 2012. Disponível em: http://www.fundaciontelefonica.com/arte_cultura/publicacioneslistado/pagina-item-publicaciones/itempubli/161/. Acesso em: 22 abr. 2018. 
AREA, Manuel y PESSOA, Teresa. De lo sólido a lo líquido: las nuevas alfabetizaciones ante los cambios culturales de la Web 2.0. Comunicar, n. 19 - v. 38, p. 13-20. 2012. Disponível em:https://www.revistacomunicar.com/verpdf.php?numero $=38$ \&articulo $=38-2012-$ o3Acesso em: 22 abr. 2018.

AVELAR MARTÍNEZ, Fernando. Estrategias de alfabetización en recursos electrónicos a través del empleo de Ebsco-Host en bibliotecas UABC, 2011. Tesis de maestría. UABC, México. Disponível em:

pedagogia.mxl.uabc.mx/.../Productos de estudiantes MD.pdf. Acesso em: 22 abr. 2018.

BAWDEN, David. Revisión de los conceptos de alfabetización informacional y alfabetización digital. Anales de Documentación, n. 5 p. 361-408, 2002. Disponível em:http://revistas.um.es/analesdoc/article/view/2261/2251Acesso em: 22 abr. 2018.

BAWDEN, David; LYN, Robinson. An intensity around information': the changing face of chemical information literacy.Journal of Information Science, v.43, n. 1, p. 1724. 2017. Disponível em:

HTTP://JOURNALS.SAGEPUB.COM/DOI/ABS/10.1177/01655.51515616919Acesso em: 22 abr. 2018.

BIELBA CALVO, Marcos; MARTÍNEZ ABAD, Fernando; HERRERA GARCÍA, María Esperanza; RODRÍGUEZ CONDE, María José. Diseño de un instrumento de evaluación de competencias informacionales en Educación Secundaria Obligatoria a través de la selección de indicadores clave. Teoría de la Educación. Educación y Cultura en la Sociedad de la Información, n. 16,v. 3,2015. Disponível em: http://campus.usal.es/ revistas trabajo/index.php/revistatesi/article/view/eks2015 163124143Acesso em: 22 abr. 2018.

BONILLA ESQUIVEL, José. Caracterización de la competencia informativa en estudiantes universitários, Tesis de doctorado. ITESO, Guadalajara. Jalisco, 2017

BONILLA, José;CONTRERAS, C.; OSUNA, Cecilia;ROBLES, A.; MARTÍNEZ, R., GUTIÉRREZ, R; TAGLIAPRIETA, Cecilia y GÁRATE, Alberto. Habilidades informativas en estudiantes del Sistema CETYS Universidad. México, 2012.

CATTS, Ralph, y LAU, Jesús. Hacia unos indicadores de Alfabetización Informacional: Marco conceptual elaborado por Ralph Catts y Jesús Lau con una lista de posibles indicadores internacionales para el suministro y el acceso a la información, y las competencias relacionadas, establecida por el Instituto de Estadística de la UNESCO. Madrid: Ministerio de cultura,2009. Disponível em: http://travesia.mcu.es/portalnb/jspui/bitstream/10421/3141/1/IndicadoresUNESC Oesp4.pdf.Acesso em: 22 abr. 2018.

DÍAZ FERNÁNDEZ, María del Pilar; VELIS MARTÍNEZ, Esther Díaz y DELGADO PÉREZ Manuel. Alfabetización Informacional digital como reclamo necesario de las sociedades científicas.Edumecentro,n. 2, v. 2, p. 42-54, 2013. Disponível em:http://www.revedumecentro.sld.cu/index.php/edumc/article/view\%20/73/149A cesso em: 22 abr. 2018. 
DOMÍNGUEZ PÉREZ, David Y OCAMPO REYES, Nicolás.Metodología para la búsqueda y selección de información en internet, una propuesta para fomentar el desarrollo de habilidades en los estudiantes de nivel superior: Curso impartido en la Universidad Insurgentes Plantel Centro,2008. Disponível em:

http://www.repositoriodigital.ipn.mx/handle/123456789/3196. Acesso em: 22 abr. 2018.

FERNÁNDEZ DE LA CRUZ, Juan Carlos. Factores de influencia en el comportamiento informativo de los docentes de tiempo completo de la Universidad Veracruzana,(Tesis de maestria), Campus Mocambo, Región Veracruz. UNAM, México, 2017.

GARCÍA LLORENTE, Héctor. Multialfabetización en la sociedad del conocimiento: competencias informacionales en el sistema educativo. Revista Lasallista de Investigación, v.12, n.2, p. 225-241. 2015. Disponível em:

http://repository.lasallista.edu.co/dspace/handle/10567/1394. Acesso em: 22 abr. 2018.

GOBIERNO FEDERAL.Ley del Instituto Nacional para la Evaluación de la Educación. México, 2013.

GONZÁLEZ NIÑO, Liliana; MARCIALES VIVAS, Gloria; CASTAÑEDA PEÑA, Harold; BARBOSA CHACÓN, Jorge y BARBOSA HERRERA, Juan. Competencia informacional: desarrollo de un instrumento para su observación. Lenguaje, v. 1, n. 41, p. 105-131, 2013. Disponível em:

http://revistalenguaje.univalle.edu.co/index.php/Lenguaje/article/view/2376. Acesso em: 22 abr. 2018.

HAMODI, Carolina; LÓPEZ-PASTOR, Víctor; LÓPEZ-PASTOR, Ana. Medios, técnicas e instrumentos de evaluación formativa y compartida del aprendizaje en educación superior.Perfiles educativos, v.37, n. 147, p. 146-161, 2015. Disponível em: http://www.scielo.org.mx/pdf/peredu/v37n147/v37n147a9.pdfAcesso em: 22 abr. 2018.

HERNÁNDEZ SAMPIERI, Roberto; COLLADO, Carlos ; BAPTISTA,Lucio. Metodología de la investigación. Mexico DF: Mc-Graw Hill, 2010.

LAGUNES DOMINGUEZ, A; Y TORRES GASTELÚ, C.Las competencias informacionales en estudiantes de nuevo ingreso en las universidades de América Latina. México, 2018. (en prensa)

LAU,Jesús;CORTÉS, Jesús. Habilidades informativas: convergencia entre ciencias de información y comunicación.Comunicar, n. 32, p. 2130,2009.https://doi.org/10.3916/c32-2009-02-001Acesso em: 22 abr. 2018.

LAU,Jesús;CORTÉS, Jesús; TARANGO, Javier; URIBE, Alejandro; MARZAL, Miguel;PIRELA, Johann; GARATE, Alberto; OSUNA, Cecilia.Demanda y competencias informativas de académicos (DECIA) una caracterización internacional: proyecto de investigación, 2015. Disponível em:

http://www.jesuslau.com.mx/decia/protocolo/index.phpAcesso em: 22 abr. 2018 
LAU, Jesús; MACHIN-MASTROMATTEO, Juan; GÁRATE, Alberto;TAGLIAPIETRAOVIES, Cecilia. Assessing Spanish-Speaking University Students' Info-Competencies with iSkills, SAILS, and an In-House Instrument: Challenges and Benefits.In European Conference on Information Literacy, n. 676, p. 327-336, 2016.

LICEA DE ARENAS, Judith. La evaluación de la alfabetización informacional. Principios, metodologías y retos. Anales de documentación, v. 10, p. 215-232, 2008. Disponível em: http://revistas.um.es/analesdoc/article/view/1161Acesso em: 22 abr. 2018

\section{LICEA DE ARENAS, Judith, GÓMEZ HERNÁNDEZ, José Antonio y VALLES}

VALENZUELA, Javier.Más sobre alfabetización informacional.Acimed, v. 20, n. 6, p. 216-227, 2009. Disponível em:http://scielo.sld.cu/pdf/aci/v20n6/acio71209.pdfAcesso em: 22 abr. 2018

MARTÍN, Carmen. Metodología de investigación en estudios de usuarios. Revista general de información y documentación, v. 17,n. 2, p. 129-149, 2007. Disponível em:

http://revistas.ucm.es/index.php/RGID/article/viewFile/RGIDo707220129A/9407. Acesso em: 22 abr. 2017.

MENESES-PLACERES, Grizly; PINTO, María. Alfinev: Modelo para evaluar la alfabetización informacional en la educación superior en Cuba. Investigación bibliotecológica, v. 25, n. 55, p. 81-106, 2011. Disponível em: http://revistas.unam.mx/index.php/ibi/article/view/32712Acesso em: 22 abr. 2017.

MINISTERIO DE EDUCACIÓN.Matriz de habilidades TIC para el aprendizaje.Santiago de Chile:2013. Disponível em:

http://www.enlaces.cl/download/matriz-de-habilidades-tic-para-elaprendizaje/Acesso em: 22 abr. 2017.

MORALES-VITELA, Thalia; LOPEZ-ORNELAS, Maricela. Diseño de un instrumento para evaluar la alfabetización informacional y su uso ético en estudiantes universitarios. En: 4to Congreso Internacional de Innovacion Educativa: 11 a 13 de Diciembre, Monterrey, Mexico, 2017, pp. 408-420.

MUÑOZ, Tomás. El cuestionario como instrumento de investigación/evaluación. Centro Universitario Santa Ana,2003. Disponível em: http://www.univsantana.com/sociologia/El Cuestionario.pdfAcesso em: 22 abr. 2017.

NOLASCO VÁZQUEZ, Pedro. La incorporación de las tecnologías de información y comunicaciones en las universidades. Estudio de caso: Universidad Veracruzana.(Tesis de doctorado) Universidad Veracruzana, México, 2017.

PEDRAZA, William. Influencia del desarrollo de competencias en información para la construcción de ante proyectos de investigación en la Escuela Agrícola Panamericana,Zamorano, (Tesis de doctorado). Universidad de Zamorano, Honduras, 2017.

PINEDA BÁEZ, Clelia; HENNIG MANZOULI, Cristina; SEGOVIA CIFUENTES, Yasbley; DÍAZ GÓMEZ, Darwin; SÁNCHEZ DUARTE, Mónica; OTERO, María y REES, Geraint. Alfabetización informacional en la educación superior virtual: logros 
y desafíos. Información, cultura y sociedad, n. 26, p. 83-104, 2012. Disponível em: http://revistascientificas.filo.uba.ar/index.php/ICS/article/view/718/696. Acesso em: 22 abr. 2018.

QUEVEDO-PACHECO, Nelva. Alfabetización informacional: aspectos esenciales. Una introducción y acercamiento sobre algunos de los aspectos más importantes de la Alfabetización Informacional (ALFIN) o competencias informativas, 2014.

Disponível em:

http://eprints.rclis.org/23091/1/Libro.ALFIN_Aspectos_Esenciales.pdfAcesso em: 22 abr. 2018.

RADCLIFF, Carolyn. Threshold achievement test for information literacy. 2017 , Online. Disponível em:http://blog.informationliteracyassessment.com/?cat=11Acesso em: 22 abr. 2018.

RODRÍGUEZ-CONDE, María; OLMOS MIGUELÁÑEZ, Susana; MARTÍNEZ ABAD, Fernando.Propiedades métricas y estructura dimensional de la adaptación española de una escala de evaluación de competencia informacional autopercibida (ILHUMASS). Revista de Investigación Educativa, v. 30, n.2, p. 347-365, 2012.DOI: http://dx.doi.org/10.6018/rie.30.2.120231. Acesso em: 22 abr. 2018

RODRÍGUEZ, David;VALLDEORIOLA, Jordi. Metodología de la investigación. México: Red Tercer Milenio. 2009. Disponível

em: http://fournier.facmed.unam.mx/deptos/seciss/images/investigacion/21.pdfAce sso em: 22 abr. 2018

SALAS CASTRO, Roberto.Factores que determinan la necesidad de solicitar asesoría informativa en usuarios universitários (Tesis de doctorado) . Universidad Veracruzana, México, 2007.

SÁNCHEZ, Marlery. Las competencias desde la perspectiva informacional: apuntes introductorios a nivel terminológico y conceptual, escenarios e iniciativas. Ciência da Informação, v. 37, n. 1, p. 107-120, 2008. Disponível em:

http://www.scielo.br/scielo.php?pid=SO10019652008000100010\&script=sci abstract\&tlng=es. Acesso em: 22 abr. 2018

KERLINGER, Fred ; LEE, Howard. Investigación del comportamiento.México: McGraw-Hill/Interamericana, 2002.

URIBE TIRADO, Alejandro; PINTO, María. 75 lecciones aprendidas en programas de alfabetización informacional en universidades iberoamericanas. Revista Española de Documentación Científica, v. 37, n. 3, p. 1-18, 2014. Disponível em:

http://dx.doi.org/10.3989/redc.2014.3.1118.

URIBE TIRADO Alejandro. Diseño, implementación y evaluación de una propuesta formativa en alfabetización informacional mediante un ambiente virtual de aprendizaje a nivel universitario:caso Escuela Interamericana de Bibliotecología Universidad de Antioquia (Tesis de Maestría).Universidad EAFIT, 2008. Disponível em: http://bibliotecadigital.udea.edu.co/dspace/handle/10495/3214Acesso em: 22 abr. 2018. 